\title{
Thermally induced structural transformations on polymorphous silicon
}

\author{
Chandana Rath \\ GRMT, Departament de Física, Universitat de Girona, Campus Montilivi, Edif. PII, E17071-Girona, \\ Catalonia, Spain; and School of Materials Science and Technology, Institute of Technology, \\ Banaras Hindu University, Varanasi, India \\ J. Farjas ${ }^{\text {a) }}$ and P. Roura \\ GRMT, Departament de Física, Universitat de Girona, Campus Montilivi, Edif. PII, E17071-Girona, \\ Catalonia, Spain \\ F. Kail and P. Roca i Cabarrocas \\ LPICM (UMR 7647 CNRS), Ecole Polytechnique, 91128 Palaiseau Cedex, France \\ E. Bertran \\ FEMAN, Departament de Física Aplicada i Optica, Universitat de Barcelona, E08028, \\ Barcelona, Catalonia, Spain
}

(Received 2 February 2005; accepted 15 June 2005)

\begin{abstract}
Polymorphous Si is a nanostructured form of hydrogenated amorphous Si that contains a small fraction of Si nanocrystals or clusters. Its thermally induced transformations such as relaxation, dehydrogenation, and crystallization have been studied by calorimetry and evolved gas analysis as a complementary technique. The observed behavior has been compared to that of conventional hydrogenated amorphous $\mathrm{Si}$ and amorphous Si nanoparticles. In the temperature range of our experiments $\left(650-700^{\circ} \mathrm{C}\right)$, crystallization takes place at almost the same temperature in polymorphous and in amorphous Si. In contrast, dehydrogenation processes reflect the presence of different hydrogen states. The calorimetry and evolved gas analysis thermograms clearly show that polymorphous $\mathrm{Si}$ shares hydrogen states of both amorphous $\mathrm{Si}$ and $\mathrm{Si}$ nanoparticles. Finally, the total energy of the main $\mathrm{Si}-\mathrm{H}$ group present in polymorphous Si has been quantified.
\end{abstract}

\section{INTRODUCTION}

Hydrogenated amorphous silicon (a-Si:H) plays a crucial role in several applications such as solar cells and flat panel displays. Because of its its technological interest, the production techniques have evolved with the aim of improving material properties (electrical conductivity, photovoltaic efficiency, stability, etc.) and deposition rate. As a result, several kinds of nanostructured silicon materials have been synthesized. The ones having the most interesting properties are microcrystalline and polymorphous silicon (pm-Si:H). Pm-Si:H is produced by plasma-enhanced chemical vapor deposition (PECVD) in a variety of plasma conditions that allow the formation of hydrogenated silicon clusters in the plasma. Before these clusters grow into powders, they are incorporated in the growing amorphous silicon film, along with $\mathrm{SiH}_{x}$ radicals.

\footnotetext{
a) Address all correspondence to this author.

e-mail: jordi.farjas@udg.es

DOI: $10.1557 / J M R .2005 .0322$
}

The result is a nanostructured material consisting of silicon clusters or nanocrystals embedded in an amorphous matrix. ${ }^{1}$ Polymorphous silicon has much better transport properties than the conventional amorphous material. ${ }^{2}$ For instance, its hole mobility, recently measured by time-of-flight experiments, ${ }^{3}$ is $2-3$ times higher. Additionally, its density of defects is one order of magnitude lower, ${ }^{4}$ which allows fabrication of p-i-n photodiodes with very low dark current densities. ${ }^{5}$ Even after light soaking, the defect density remains lower than in conventional a-Si:H. ${ }^{2}$ Consequently, enhanced photovoltaic conversion efficiency is expected. ${ }^{6}$

These properties indicate that the structure of pm-Si:H may be quite different from that of a-Si:H. In fact, highresolution transmission electron microscopy ${ }^{1}$ and fluctuation electron microscopy ${ }^{7}$ have revealed a higher degree of medium range order indicating that $\mathrm{pm}-\mathrm{Si}: \mathrm{H}$ is a more relaxed material than conventional amorphous silicon. On the other hand, infrared spectroscopy (IR) as well as evolved gas analysis experiments (EGA) show the existence of hydrogen states not detected previously in a-Si:H. ${ }^{8,9}$ All these features should have an effect on 
the energy of pm-Si:H that could be quantified by calorimetric measurements.

In this paper, we analyze by differential scanning calorimetry (DSC) the heat exchanged during the structural transformations that take place in $\mathrm{pm}-\mathrm{Si}: \mathrm{H}$ when the material is heated at constant rate. In addition to the enthalpy of the transformations, the thermal signal can give complementary information concerning their kinetics. This is of special interest in the case of hydrogen desorption because the EGA thermograms used to characterize its kinetics are highly influenced by the hydrogen diffusion, ${ }^{10}$ and the main step involving the breaking and rearrangement of bonds remains hidden. Despite the possibilities that offer the calorimetric techniques, they have seldom been used for the study of hydrogenated amorphous materials.

\section{EXPERIMENTAL}

Three different materials have been prepared for this study: conventional hydrogenated amorphous (a-Si:H; our ref. 310051) and polymorphous (pm-Si:H; our ref. 310032) silicon films and hydrogenated amorphous silicon nanoparticles (our ref. 190598) in a PECVD system by radio frequency (rf) glow discharge. a-Si:H and $\mathrm{Si}$ nanoparticles were obtained by decomposition of pure silane, whereas in the case of $\mathrm{pm}-\mathrm{Si}: \mathrm{H}$, silane was diluted to $2 \%$ in $\mathrm{H}_{2}$. Deposition time was as long as $10 \mathrm{~h}$ for the films, which resulted in thickness of around $10 \mu \mathrm{m}$. No substrate was used, and the material consisted of flakes peeling from the inner surfaces of the reactor chamber, which was maintained at $250{ }^{\circ} \mathrm{C}$ for the films and at room temperature for the nanoparticles. The chemical composition was quantified by elementary analysis. In this technique, the mass fraction of hydrogen is determined by the amount of $\mathrm{H}_{2} \mathrm{O}$ obtained after complete combustion of the sample. For the films, this information was readily converted into the atomic fraction: $\mathrm{Si}_{1} \mathrm{H}_{0.12}$ for a-Si:H and $\mathrm{Si}_{1} \mathrm{H}_{0.16}$ for pm-Si:H. For the nanoparticles, the procedure was slightly longer because they contain, in the as-grown state, a small amount of oxygen that has to be quantified. We have obtained it from the sample mass gain measured after complete oxidation in a thermobalance. The atomic composition of nanoparticles is $\mathrm{Si}_{1} \mathrm{H}_{0.35} \mathrm{O}_{0.3}$.

Calorimetric measurements were carried out in a DSC in inert atmosphere of Ar. To minimize residual oxidation at high temperature, for the experiments with $\mathrm{Si}$ nanoparticles, the Ar flow rate was much higher (300 $\mathrm{ml} / \mathrm{min}$ ) than with the other materials $(40 \mathrm{ml} / \mathrm{min})$. The thermal signal due to dehydrogenation and relaxation processes was measured during rapid heating ramps (40 K/min); otherwise it was difficult to distinguish the signal from the baseline. On the other hand, the crystallization peak was detected at a much lower heating rate of $2.5 \mathrm{~K} / \mathrm{min}$. Because of its thermal activation kinetics, at $40 \mathrm{~K} / \mathrm{min}$, this process would take place above the maximum temperature allowed in our DSC apparatus $\left(700{ }^{\circ} \mathrm{C}\right)$. After any heating ramp up to $700{ }^{\circ} \mathrm{C}$, the sample was held at $670{ }^{\circ} \mathrm{C}$ for half an hour to ensure that in a second heating ramp, no structural transformation takes place. During the second heating ramp, the sample is, in fact, inert, and the corresponding thermogram can be taken as the signal baseline. Thus, the DSC thermograms shown in this paper were obtained by subtracting the curves of the second and first heating ramps. The temperature and heat flow were corrected by proper calibration at the experimental conditions.

Evolved gas analyses were performed in a vacuum furnace pumped down to $10^{-4} \mathrm{~Pa}$ by a turbomolecular pump. The evolved hydrogen atoms were detected by a mass spectrometer whose signal was considered, as usual, ${ }^{11}$ proportional to the evolution rate. To allow comparison with DSC results, the EGA experiments were done at $40 \mathrm{~K} / \mathrm{min}$, too. Complementary experiments have shown that the delay between hydrogen evolution from the sample and detection is negligible, as expected.

\section{RESULTS}

The heat released from $\mathrm{pm}-\mathrm{Si}: \mathrm{H}$ during a heating ramp at $40 \mathrm{~K} / \mathrm{min}$ is shown in the solid curve of Fig. 1. An exothermic process begins with a threshold located between 250 and $300{ }^{\circ} \mathrm{C}$ and continues up to $700{ }^{\circ} \mathrm{C}$. Three peaks $\mathrm{A}, \mathrm{B}$, and $\mathrm{C}^{\prime}$ can be clearly distinguished with maxima at 390,460 , and $580{ }^{\circ} \mathrm{C}$, respectively. Above

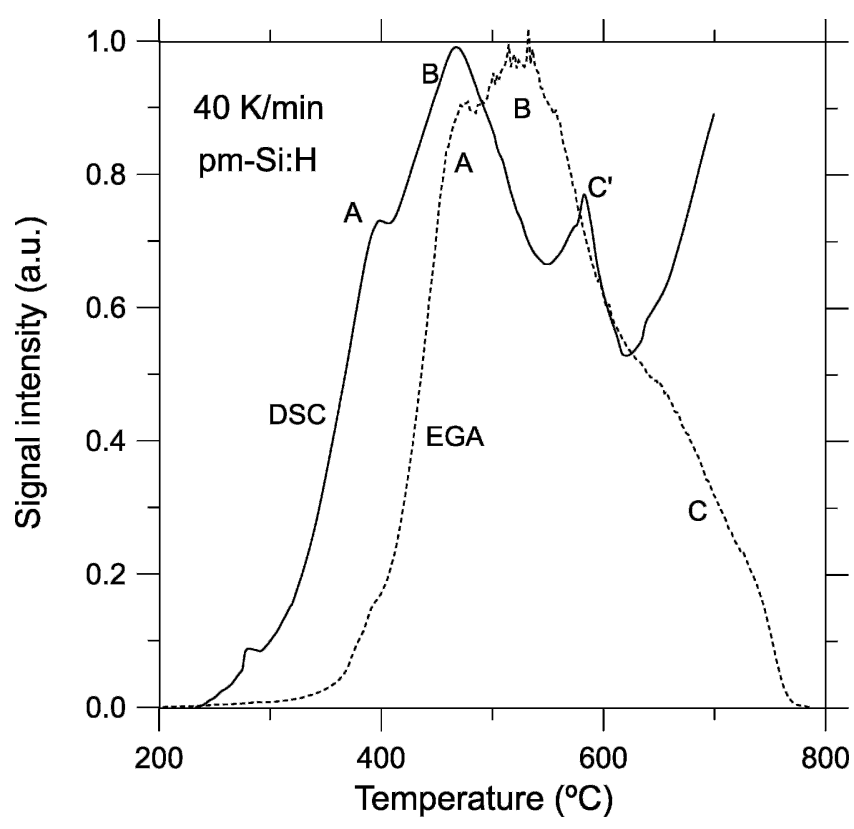

FIG. 1. Differential scanning calorimetry and evolved hydrogen analysis thermograms measured on polymorphous silicon (the small peak at $290{ }^{\circ} \mathrm{C}$ is an artifact). 
$620{ }^{\circ} \mathrm{C}$, the signal increases steadily, indicating that another exothermic process continues above the maximum temperature allowed in our DSC $\left(700{ }^{\circ} \mathrm{C}\right)$. In fact, when the heating ramp is programmed at a much lower rate $(2.5 \mathrm{~K} / \mathrm{min})$, this process reaches completion within the DSC temperature range. In the corresponding thermogram, a peak appears between 650 and $700{ }^{\circ} \mathrm{C}$ (inset of Fig. 2). The peak area delivers the enthalpy of the process $(12.5 \pm 0.5 \mathrm{KJ} / \mathrm{Si} \mathrm{mol})$, which matches the published values of crystallization enthalpy ${ }^{12}$ of a-Si $(11.9 \pm$ $0.7 \mathrm{KJ} / \mathrm{Si} \mathrm{mol}$ ). The heat evolved before crystallization (Fig. 1) is much lower and can be approximately quantified through the area of the thermogram at $40 \mathrm{~K} / \mathrm{min}$. The enthalpy of these low temperature processes is $180 \pm 20 \mathrm{~J} / \mathrm{g}$. The structural transformations related to these processes will be analyzed after comparison with complementary DSC and EGA experiments.

The dashed curve in Fig.1 represents the evolved hydrogen and thus corresponds to the rate at which hydrogen atoms reach the external surface of the sample and leave it. Leaving apart the contribution of crystallization to the DSC thermogram, the EGA curve is a somewhat smoothed version of the DSC curve, and it is shifted due to hydrogen diffusion. Three main features A, B, and C are also present. Consequently, at this stage we can state that the peaks A, B, and $\mathrm{C}^{\prime}$ in the DSC curve are related to dehydrogenation processes and, presumably, to different hydrogen states.

Although the relationship between the peaks observed in DSC and dehydrogenation is established, the question

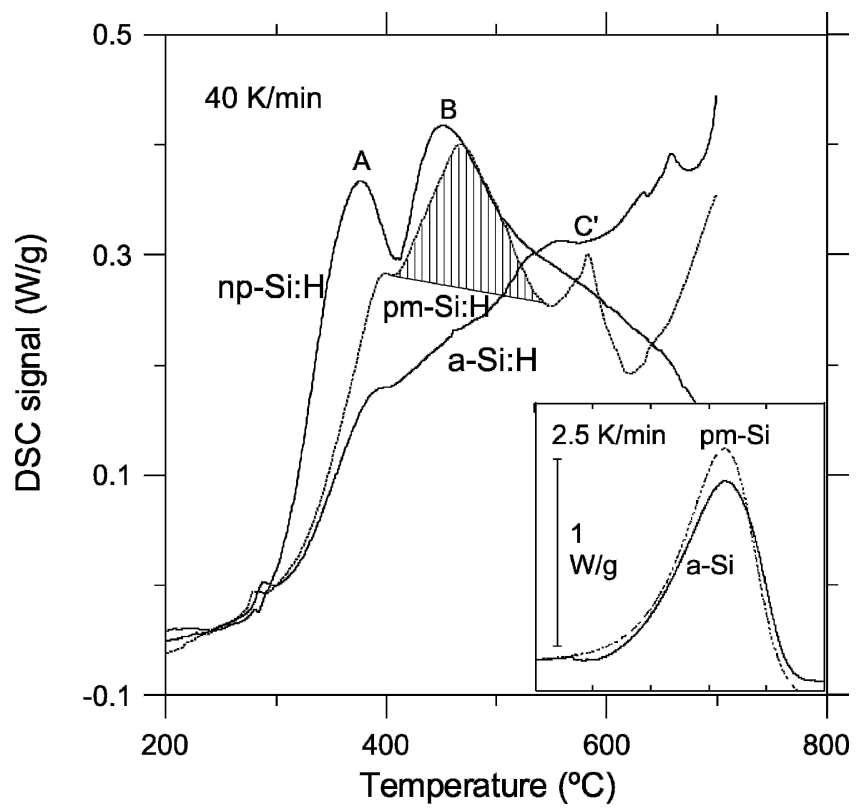

FIG. 2. Differential scanning calorimetry thermograms measured on three forms of hydrogenated amorphous silicon: conventional (a-Si:H), polymorphous (pm-Si:H), and nanoparticles (np-Si). The significance of the shaded area is explained in the text. (inset) Crystallization peaks of a-Si:H and pm-Si:H. remains whether there exists another structural transformation contributing to the thermal signal. This question can be analyzed after looking at the DSC and EGA thermograms measured in the other two materials (a-Si:H and $\mathrm{Si}$ nanoparticles) under the same conditions. Concerning the DSC curves (Fig. 2), it is worth noting that the low temperature threshold is common for all three materials. The main differences with respect to the pm$\mathrm{Si}: \mathrm{H}$ are the following: (i) in $\mathrm{Si}$ nanoparticles, the A feature is more intense resulting in a well resolved peak and (ii) peak B is not detected in a-Si:H. These differences are also present in the corresponding EGA curves (Fig. 3), where (i) peak A is very prominent in Si nanoparticles and (ii) peak B is almost absent in a-Si:H. This correspondence between EGA and DSC curves for all three materials reinforces the conclusion that features A and $\mathrm{B}$ in the thermal signal of $\mathrm{pm}-\mathrm{Si}: \mathrm{H}$ correspond to dehydrogenation processes (the case of peak $\mathrm{C}^{\prime}$ will be discussed below). On the other hand, we can now state that dehydrogenation is not the only process contributing to the thermal signal. The dehydrogenation rate in a-Si:H begins to be significant at about $500{ }^{\circ} \mathrm{C}$ while the threshold in its DSC curve (Fig. 2) is located at $300{ }^{\circ} \mathrm{C}$. Thus, the heat evolved in a-Si:H below $500{ }^{\circ} \mathrm{C}$ can hardly be assigned to dehydrogenation. Instead, it is related to the so-called "relaxation processes," which, in nonhydrogenated amorphous $\mathrm{Si}$, are usually attributed to a diminution of the internal stress of the amorphous $\mathrm{Si}$ network. ${ }^{13}$ Due to the coincidence of the DSC low temperature threshold for a-Si and $\mathrm{pm}-\mathrm{Si}: \mathrm{H}$, we conclude that the DSC signal of pm-Si:H can be understood as the

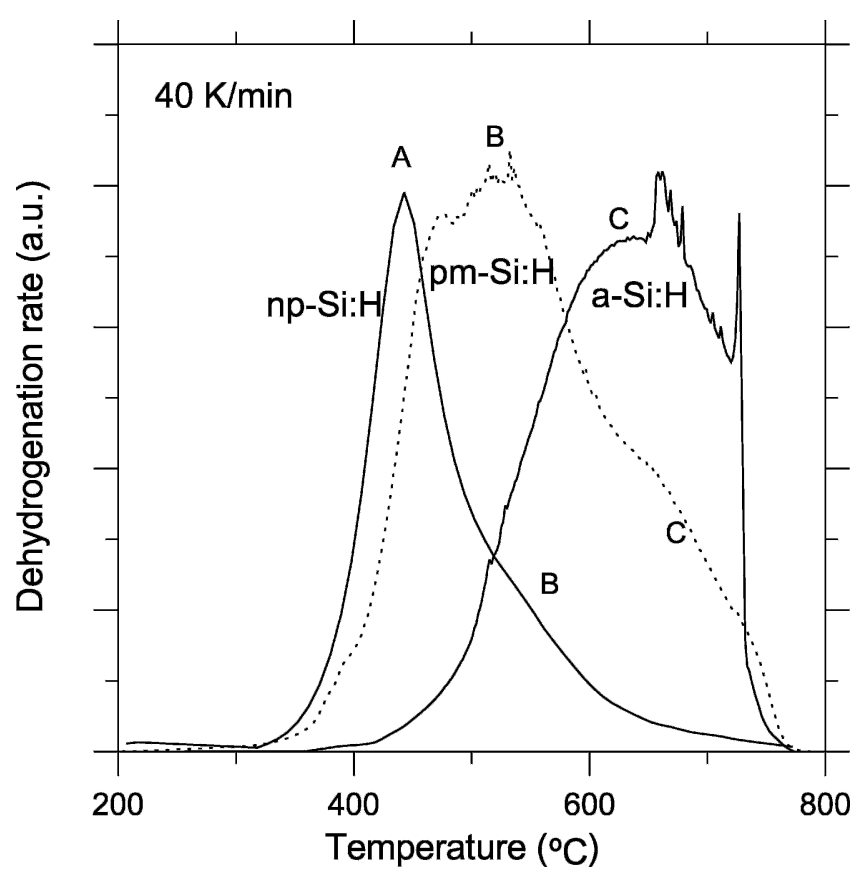

FIG. 3. Evolved gas thermograms of hydrogen measured on the three materials. 
addition of several exothermic peaks (related to dehydrogenation) to the more continuous signal related to structural relaxation (similar to the one observed on nonhydrogenated amorphous Silicon ${ }^{13}$ ). This conclusion is reinforced by DSC thermograms measured at several heating rates. ${ }^{14}$

Finally, we performed DSC experiments at a lower heating rate $(2.5 \mathrm{~K} / \mathrm{min})$ for $\mathrm{Si}$ nanoparticles and a-Si:H, too. The corresponding thermogram of a-Si:H is shown in the inset of Fig. 2. The crystallization peak of a-Si coincides with that of $\mathrm{pm}-\mathrm{Si}: \mathrm{H}$, whereas this process occurs at higher temperature in $\mathrm{Si}$ nanoparticles ${ }^{15}$ (not shown in the figure). In Ref. 14, it was shown that, at low temperature $\left(590{ }^{\circ} \mathrm{C}\right)$ and due to the presence of small crystallites, pm-Si:H crystallizes faster than a-Si:H does. This apparent contradiction with previous results will be discussed in next section.

\section{ANALYSIS}

\section{A. Hydrogen states}

To elucidate which are the hydrogen states in pm-Si:H, the analysis will rely on what is known in the other two materials.

In a-Si:H, the EGA thermogram contains the single band, $\mathrm{C}$. We think that the abrupt change in the desorption rate observed at $650{ }^{\circ} \mathrm{C}$ is due to some kind of mechanical disruption of the material, caused by molecular hydrogen trapped in it, and that the last sharp peak is induced by crystallization. The main band $\mathrm{C}$ has been well characterized and is known to correspond to isolated $\mathrm{Si}-\mathrm{H}$ groups in the bulk. This dominates the desorption curves of device-quality a-Si:H materials. ${ }^{10}$ The IR stretching bands of these materials contain one single peak ${ }^{17}$ centered at $2000 \mathrm{~cm}^{-1}$. This state of hydrogen is present in $\mathrm{pm}-\mathrm{Si}: \mathrm{H}$ and is responsible for the shoulder that appears in its EGA thermogram (Fig. 3) above $600{ }^{\circ} \mathrm{C}$.

In Si nanoparticles, the EGA thermogram is dominated by peak A. It is also observed in a-Si:H films grown at low temperature and corresponds to the desorption of hydrogen atoms that are bonded to the surfaces of internal voids. ${ }^{17}$ This assignment is supported by the observation of an intense IR component at $2080 \mathrm{~cm}^{-1}$ in addition to the usual peak ${ }^{18}$ at $2000 \mathrm{~cm}^{-1}$. Although the state of our samples (in the form of pellets) makes it impossible to obtain reliable IR spectra, systematic IR experiments on $\mathrm{pm}-\mathrm{Si}: \mathrm{H}$ thin films ${ }^{9}$ have shown that this state of hydrogen is present in pm-Si:H, too. It is related to feature A of the EGA thermogram (Fig. 1). Thus, from the comparison with $\mathrm{Si}$-nanoparticles we can say that peak $\mathrm{A}$ is related to $\mathrm{H}$ in voids more or less connected to the external surfaces of the material.

Finally, the origin of the desorption process B, which dominates the EGA thermogram of pm-Si:H, should be explained. This process is detected as a higher temperature shoulder in Si nanoparticles and is almost undetectable in a-Si:H. Independent studies ${ }^{8,19}$ have shown that it dominates the EGA thermogram of microcrystalline silicon. It is correlated with the existence in the IR spectra of a component in the stretching region centered at $2030 \mathrm{~cm}^{-1}$ and a vibrational mode in the wagging region at $873 \mathrm{~cm}^{-1}$, both absent in conventional a-Si:H. ${ }^{8,9}$ Its vibrational energy, intermediate between the $\mathrm{Si}-\mathrm{H}$ groups in bulk $\left(2000 \mathrm{~cm}^{-1}\right)$ and $\mathrm{Si}-\mathrm{H}$ groups in surfaces $\left(2080 \mathrm{~cm}^{-1}\right)$, as well as its presence in microcrystalline silicon, ${ }^{19,20}$ has led us propose that it corresponds to hydrogen bonded on the surfaces of nanoparticles or silicon clusters. In fact, the plasma conditions of pm-Si:H are such that, in addition to $\mathrm{SiH}_{x}$ radicals, clusters do contribute to the film growth. These clusters are then embedded in the amorphous matrix, and the extra vibrational mode would correspond to hydrogen located at the interface between both phases.

We conclude this analysis with an approximate quantification of the amount of the different kinds of Si-H groups in pm-Si:H. A fit of the EGA thermogram with a number of gaussian components leads to the following values: $n_{\mathrm{A}} / n_{\mathrm{Si}}=0.019(\mathrm{H}$ on surfaces $), n_{\mathrm{B}} / n_{\mathrm{Si}}=0.11$ ( $\mathrm{H}$ on interfaces), and $n_{\mathrm{C}} / n_{\mathrm{Si}}=0.038$ ( $\mathrm{Si}-\mathrm{H}$ isolated groups).

\section{B. Enthalpy of dehydrogenation}

Given that the heat evolved before crystallization has contributions from both dehydrogenation and structural relaxation, it is difficult, in general, to quantify the enthalpy of dehydrogenation of each $\mathrm{Si}-\mathrm{H}$ group present in pm-Si:H. The feature A in the DSC thermogram of pm$\mathrm{Si}: \mathrm{H}$ has probably a more important contribution from structural relaxation because its shape is almost identical to that of the corresponding signal in a-Si:H (for which dehydrogenation does not occur at this low temperature). On the other hand, the assignment of peak $\mathrm{C}^{\prime}$ of the pm-Si:H DSC curve to the process of desorption of $\mathrm{H}$ coming from isolated groups (shoulder $\mathrm{C}$ in the EGA curve) is not certain. In fact, if this were the case, one should expect a more pronounced peak at the same position in the DSC thermogram of a-Si:H. Instead, a smoother and less intense band can be distinguished over the continuous signal at a lower temperature (around $550{ }^{\circ} \mathrm{C}$ ).

The only process whose enthalpy can be quantified with confidence is that corresponding to peak $\mathrm{B}(\mathrm{H}$ in interfaces). We consider that the heat evolved in this process corresponds to the shaded area below the B peak indicated in Fig. 2: $13.6 \pm 1.0 \mathrm{~J} / \mathrm{g}$. Now, as the number of hydrogen atoms in this state has already been quantified $\left(n_{\mathrm{B}} / n_{\mathrm{Si}}=0.11\right)$, the enthalpy of the process.

$$
\mathrm{Si}-\mathrm{H}\left(\text { state B) } \rightarrow \mathrm{Si}+1 / 2 \mathrm{H}_{2},\right.
$$


where no dangling bonds are left behind, is $3.2 \pm$ $0.6 \mathrm{KJ} / \mathrm{mol}$ of $\mathrm{H}$ or, equivalently, $33 \pm 6 \mathrm{meV}$ per $\mathrm{H}$ atom. This value is similar to the energy published in a previous paper devoted to nanoparticles $(30 \pm 10 \mathrm{meV} / \mathrm{H}$ atom). ${ }^{18}$ In the same paper, the measured energy of $\mathrm{H}$ in internal voids (peak A) was similar $(40-90 \mathrm{meV} / \mathrm{H}$ atom). As far as we know, these are the only reliable quantifications of the enthalpy of dehydrogenation in amorphous silicon materials.

Few theoretical calculations of the energies of $\mathrm{Si}-\mathrm{H}$ groups are available. Among them, Allan et al. ${ }^{21}$ predicted that dehydrogenation in a-Si:H would be endothermic with enthalpies around $-100 \mathrm{MeV} / \mathrm{H}$ atom whereas van der Walle ${ }^{22}$ predicted an exothermic energy of around $150 \mathrm{MeV} / \mathrm{H}$ atom. Our experimental value is midway and confirms the result of both authors in the sense that energies of hydrogen groups are close to zero when compared with the energies of the individual bonds that are broken or formed during dehydrogenation. For instance, the binding energy of a $\mathrm{H}_{2}$ molecule is of $2.26 \mathrm{eV}$ per $\mathrm{H}$ atom. ${ }^{23}$ From a qualitative point of view, our results clearly indicate that dehydrogenation of $\mathrm{H}$ from surfaces and interfaces is an exothermic process. This is a result that we have obtained for any particular kind of hydrogenated silicon material and with different commercial apparatus. In our paper devoted to nanoparticles, ${ }^{18}$ we discarded any significant disturbance of the DSC sensitivity by the $\mathrm{H}_{2}$-rich atmosphere created in the furnace during dehydrogenation. The present experiments discard the possibility that parasitic oxidation (exothermic) could have on the sign of the B-peak because, in contras t to nanoparticles, $\mathrm{pm}-\mathrm{Si}: \mathrm{H}$ has a much denser microstructure, and thus it is protected against oxidation. In fact, even in air, no mass gain has been recorded after a heating ramp up to $700{ }^{\circ} \mathrm{C}$. In view of our results, and in agreement with the analyses given by other authors, ${ }^{24}$ we consider that those thermograms exhibiting endothermic dehydrogenation peaks ${ }^{25-27}$ should be kept under suspicion.

\section{Crystallization}

A detailed analysis of ellipsometry experiments ${ }^{28}$ has delivered a density of nanocrystallites in pm-Si:H that corresponds to a crystalline fraction ranging from $0 \%$ to $8 \%$, the particular value depending on the deposition conditions. Consequently, one would expect a lower crystallization enthalpy in pm-Si:H than in a-Si:H. The maximum diminution would be $0.9 \mathrm{KJ} / \mathrm{Si}$ mol for a crystalline fraction of $8 \%$. The fact is that the experimental enthalpy of $12.5 \pm 0.5$ falls in the upper range of the published $^{12}$ values for a-Si:H $(11.9 \pm 0.7 \mathrm{KJ} / \mathrm{Si} \mathrm{mol})$. Our own experiment on a-Si:H delivered an enthalpy of $10.9 \pm 0.5$, which is clearly lower than that for pm-Si:H. We conclude that it is difficult to quantify a small crystalline fraction by calorimetry. This is because, in contrast to solidification, crystallization is not a well defined thermodynamic transition. The crystallization enthalpy varies with the degree of relaxation of the amorphous phase at the moment it crystallizes, so it depends on the thermal history.

Concerning kinetics, the coincidence between amorphous and polymorphous $\mathrm{Si}$ is striking. The peak temperature of $682{ }^{\circ} \mathrm{C}$ at $2.5 \mathrm{~K} / \mathrm{min}$ corresponds to the expected value for three-dimensional crystal growth and homogeneous nucleation. ${ }^{29}$ This means that, at the experimental conditions, the particular microstructure of pm-Si:H (clusters or nanoparticles embedded in an amorphous matrix) does not have any effect on the nucleation rate. This is in contrast to previous crystallization experiments carried out at a lower temperature $\left(590^{\circ} \mathrm{C}\right)$ during isothermal treatments, where $\mathrm{pm}-\mathrm{Si}: \mathrm{H}$ crystallized faster than a-Si:H did. ${ }^{16}$ In that case, nucleation was heterogeneous, and the grain size after crystallization was correlated with the density of nanoparticles revealed by transmission electron microscopy. This apparent contradiction could be understood if we take into account the pronounced dependence of the homogeneous nucleation rate $r_{\mathrm{n}}$ on temperature. ${ }^{30}$ At $590{ }^{\circ} \mathrm{C}, r_{\mathrm{n}}$ is 110 times lower than at $682{ }^{\circ} \mathrm{C}$. Consequently, heterogeneous nucleation may dominate over homogeneous nucleation at low temperature whereas the opposite may hold at high temperature.

\section{CONCLUSIONS}

In this paper, we have reported calorimetric experiments carried out on polymorphous silicon. In the thermograms, three different structural processes can be distinguished: (i) structural relaxation that gives rise to a continuous heat release from the signal threshold at $300{ }^{\circ} \mathrm{C}$ up to the maximum temperature of the experiments; (ii) dehydrogenation, which is related to slightly exothermic peaks superimposed to the signal of relaxation, and (iii) crystallization.

Dehydrogenation was monitored through the thermal signal (differential calorimetry) and the flux of atoms leaving the sample. Comparison with similar experiments carried out on conventional dehydrogenated amorphous $\mathrm{Si}$ and a-Si nanoparticles allowed us to identify the main $\mathrm{Si}-\mathrm{H}$ groups present in polymorphous silicon. Most of the $\mathrm{H}$ atoms were bonded in interfaces, whereas a smaller fraction was located in internal voids or diluted as $\mathrm{Si}-\mathrm{H}$ isolated groups in the bulk. The thermal peak corresponding to dehydrogenation of $\mathrm{H}$ located in interfaces was well resolved over relaxation and allowed us to quantify the corresponding enthalpy, which was found to be $30 \mathrm{MeV} / \mathrm{H}$ atom.

Additionally, crystallization of polymorphous silicon was analyzed by calorimetry and compared with conventional hydrogenated amorphous silicon. At the experimental conditions, the particular microstructure of 
polymorphous silicon, with clusters and nanocrystallites embedded in an amorphous matrix, does not have any substantial effect on the crystallization enthalpy nor on its kinetics.

While these studies might seem far away from the optimization of devices based on pm-Si:H thin films, we think that further device optimization can only be achieved through a detailed understanding of the structure of such complex materials. Indeed, the film structure and hydrogen bonding are key parameters for designing materials more stable against metastable changes such as the Staebler Wronski effect.

\section{ACKNOWLEDGMENTS}

This work was supported by the Spanish Programa Nacional de Materiales under Contract Nos. MAT-200204236-C04-01 and -02. One of the authors (C. Rath) wishes to acknowledge the Ministerio de Ciencia y Tecnología, Government of Spain, for support.

\section{REFERENCES}

1. A. Fontcuberta, I. Morral, H. Hofmeister, and P. Roca i Cabarrocas: Structure of plasma-deposited polymorphous silicon. J. NonCryst. Solids 299-302, 284 (2002).

2. R. Butté, R. Meaudre, M. Meaudre, S. Vignoli, C. Longeaud, J.P. Keider, and P. Roca i Cabarrocas: Some electronic and metastability properties of a new nanostructured material: Hydrogenated polymorphous silicon. Philos. Mag. B 79, 1079 (1999).

3. M. Brinza, G. Adriaensens, and P. Roca i Cabarrocas: Time-offlight measurements of carrier drift mobilities in polymorphous silicon. Thin Solid Films 427, 123 (2003).

4. J.P. Kleider, C. Longeaud, M. Gauthier, M. Meaudre, R. Meaudre, R. Butté, S. Vignoli, and P. Roca i Cabarrocas: Very low densities of localized states at the Fermi level in hydrogenated polymorphous silicon from capacitance and space-charge-limited current measurements. Appl. Phys. Lett. 75, 3351 (1999).

5. S. Tchakarov, P. Roca i Cabarrocas, U. Dutta, P. Chatterjee, and E. Equer: Experimental study and modeling of reverse-bias dark currents in PIN structures using amorphous and polymorphous silicon. J. Appl. Phys. 94, 7317 (2003).

6. Y. Poissant, P. Chatterjee, and P. Roca i Cabarrocas: Analysis and optimization of the performance of polymorphous silicon solar cells: Experimental characterization and computer modeling. J. Appl. Phys. 94, 7305 (2003).

7. P.M. Voyles and J.R. Abelson: Medium-range order in amorphous silicon measured by fluctuation electron microscopy. Sol. Energy Mater. Sol. Cells 78, 85 (2003).

8. S. Lebib and P. Roca i Cabarrocas: Structure and hydrogen bonding in plasma deposited polymorphous silicon thin films. Eur. Phys. J. AP 26, 17 (2004).

9. R. Meaudre, R. Butté, S. Vignoli, M. Meaundre, L. Saviot, O. Marty, and P. Roca i Cabarrocas: Structural properties and recombination processes in hydrogenated polymorphous silicon. Eur. Phys. J. 22, 171 (2003).

10. W. Beyer and A. Wagner: Determination of the hydrogen diffusion-coefficient in hydrogenated amorphous-silicon from hydrogen effusion experiments. J. Appl. Phys. 53, 8745 (1982).
11. X. Jiang, W. Beyer, and K. Reichelt: Gas evolution from hydrogenated amorphous-carbon films. J. Appl. Phys. 68, 1378 (1990).

12. E.P. Donovan, F. Spaepen, D. Turnbull, J.M. Poate, and D.C. Jacobson: Heat of crystallization and melting point of amorphous silicon. Appl. Phys. Lett. 42, 698 (1983).

13. W.C. Sinke, S. Roorda, and F.W. Saris: Variable strain-energy in amorphous-silicon. J. Mater. Res. 3, 1201 (1988).

14. P. Roura and J. Farjas (unpublished).

15. J. Farjas, J. Serra-Miralles, P. Roura, P. Roca i Cabarrocas, and E. Bertran: Anomalous crystallization kinetics of a-Si:H at high heating rates. J. Mater. Res. 20, 277 (2005).

16. E. Bertran, S.N. Sharma, G. Viera, J. Costa, P. St'ahel, and P. Roca i Cabarrocas: Effect of the nanoparticles on the structure and crystallization of amorphous silicon thin films produced by $\mathrm{rf}$ glow discharge. J. Mater. Res. 13, 2476 (1998).

17. K. Zellama, L. Chahed, P. Sládek, M.L. Thèye, J.H. von Barbeleben, and P. Roca i Cabarrocas: Hydrogen-effusion-induced structural changes and defects in a-Si:H films: Dependence upon the film microstructure. Phys. Rev. B 53, 3804 (1996).

18. J. Farjas, D. Das, J. Fort, P. Roura, and E. Bertran: Calorimetry of hydrogen desorption from a-Si nanoparticles. Phys. Rev. B 65, 115403 (2002).

19. S. Lebib and P. Roca i Cabarrocas: Effects of ion energy on the crystal size and hydrogen bonding in plasma-deposited nanocrystalline silicon thin films. J. Appl. Phys. 97, 104334 (2005).

20. P. Roca i Cabarrocas, S. Hamma, S.N. Sharma, G. Viera, E. Bertran, and J. Costa: Nanoparticle formation in low-pressure silane plasmas: Bridging the gap between a-Si:H and microcrystalline Si films. J. Non-Cryst. Solids 227-230, 871 (1998).

21. D.C. Allan, J.D. Joannopoulos, and W.B. Pollard: Electronic states and total energies in hydrogenated amorphous-silicon. Phys. Rev. B 25, 1065 (1982).

22. C.G. van der Walle: Energies of various configurations of hydrogen in silicon. Phys. Rev. B 49, 4579 (1994).

23. CRC Handbook of Chemistry and Physics, 81st ed. (CRC Press, Boca Raton, FL, 2001).

24. L. Battezzati, F. Demichelis, C.F. Pirri, and L. Tresso: Effects of temperature on structural-properties of hydrogenated amorphous silicon-germanium and carbon-silicon-germanium alloys. J. Appl. Phys. 69, 2029 (1991).

25. P. Delli Veneri, C. Privato, and E. Terzini: Changes of hydrogen evolution thermodynamics induced by $\mathrm{He}$ and $\mathrm{H}-2$ dilution in PECVD a-Si:H films: Influence on thermal crystallization. J. NonCryst. Solids 266-269, 635 (2000).

26. L. Battezzati, F. Demichelis, C.F. Pirri, A. Tagliaferro, and E. Tresso: Investigation on structural-changes in amorphous tetrahedral alloys by means of differential scanning calorimetry. J. Non-Cryst. Solids 137-138, 87 (1991).

27. S.M. Lee, S.J. Jones, Y-M. Li, W.A. Turner, and W. Paul: Comparison of the structural-properties of a-Si-H prepared from $\mathrm{SiH}_{4}$ and $\mathrm{SiH}_{4}+\mathrm{H}_{2}$ plasmas, and correlation of the structure with the photoelectronic properties. Philos. Mag. B 60, 547 (1989).

28. A. Fontcuberta, I. Morral, P. Roca i Cabarrocas, and C. Clerc: Structure and hydrogen content of polymorphous silicon thin films studied by spectroscopic ellipsometry and nuclear measurements. Phys. Rev. B 69, 125307 (2004).

29. J. Farjas, P. Chandana, R. Roura, and P. Roca i Cabarrocas: Crystallization kinetics of hydrogenated amorphous silicon thick films grown by plasma-enhanced chemical vapour deposition. Appl. Surf. Sci. 238, 165 (2004).

30. C. Spinella, S. Lombardo, and F. Priolo: Crystal grain nucleation in amorphous silicon. J. Appl. Phys. 84, 5383 (1998). 